EPJ Web of Conferences 81, 06010 (2014)

DOI: $10.1051 /$ epjconf/ 20148106010

(C) Owned by the authors, published by EDP Sciences, 2014

\title{
Muon induced deuteron disintegration in three-dimensions
}

\author{
Kacper Topolnicki ${ }^{1, a}$, Jacek Golak ${ }^{1}$, Roman Skibiński ${ }^{1}$, Laura Elisa Marcucci ${ }^{2}$, Henryk Witała ${ }^{1}$, \\ and Alaa Eldeen Elmeshneb ${ }^{1}$ \\ ${ }^{1}$ M. Smoluchowski Institute of Physics, Jagiellonian University, Kraków, Poland \\ ${ }^{2}$ Department of Physics, University of Pisa, Pisa, Italy
}

\begin{abstract}
We present a three-dimensional (3D) description of muon induced deuteron disintegration. This reaction is treated as the decay of the muonic atom with the muon initially on the lowest $K$ shell. Our aim is to calculate the total and differential decay rates. We work in momentum space and use 3D momentum eigenstates directly. This approach allowed us to calculate the appropriate nuclear matrix elements, necessary building blocks for the differential decay rate, in a single step. For contrast - in classical calculations many partial-waves have to be taken into account. We achieved a very good agreement between the 3D and partial-wave methods for calculations that involve single-nucleon currents. Our result for the total decay rate is also in agreement with experimental values, though these are not very precise. This success motivates us to also include two-nucleon current contributions that include the meson exchange currents. Additionally, our formalism can also be applied to other, so far poorly described, processes like: $\mu+{ }^{3} \mathrm{He} \rightarrow v+n+d$ or $\mu+{ }^{3} \mathrm{He} \rightarrow v+n+n+p$.
\end{abstract}

\section{Introduction}

Muon induced deuteron disintegration is presented in a 3D formalism, that instead of partial wave decomposition of operators involved in the calculations, uses the 3D momentum eigenstates of the nucleon directly. This formalism was successfully applied to transition operator calculations in [1] and to the description of electron induced deuteron disintegration in [2]. This short paper aims at show the advantages of this method by underlining the direct approach that it makes possible.

\section{Formal ingredients}

The kinematic situation is presented in Figure 1. Initially the muon is captured by the deuteron nucleus temporarily creating a muonic atom with the muon on the lowest $K$ shell. In the final state the atom disintegrates into two neutrons and a muonic neutrino. The calculation outlined below aims to arrive at the decay rate of the muonic atom, a more complete description can be found in [3].

The reaction is described using the Fermi approximation (for example [4]) and we consider the case with the muon spin coupled to the deuteron angular momentum to form a hyperfine doublet

\footnotetext{
a e-mail: kacper.topolnicki@uj.edu.pl
} 

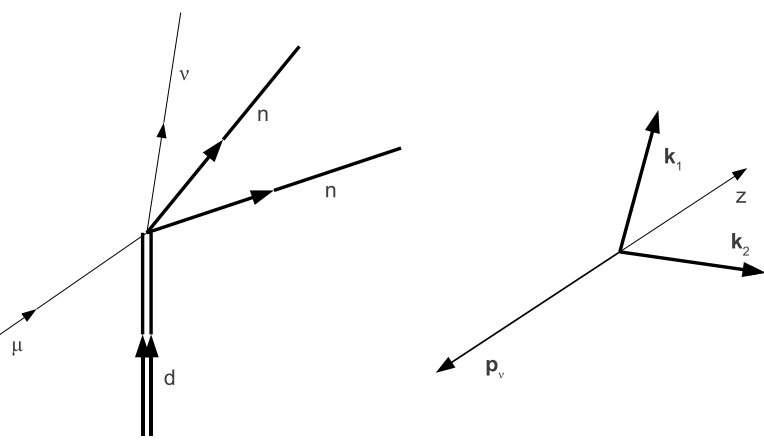

Figure 1: Diagrammatic representation and kinematics for the $\mu+d \rightarrow v_{\mu}+n+n$ reaction. This process is treated as the decay of the muonic atom, with the muon on the $K$ shell.

$f=1 / 2$ and quadruplet $f=3 / 2$, separately. The differential decay rate is governed by the weak transition operator $T_{w} \propto L_{\lambda} J^{\lambda}$ where:

$$
L_{\lambda}=\frac{1}{(2 \pi)^{3}} \bar{u}\left(\boldsymbol{p}_{v}, s_{v}\right) \gamma_{\lambda}\left(1-\gamma_{5}\right) u\left(\boldsymbol{p}_{\mu}, s_{\mu}\right)
$$

is the leptonic part and

$$
J^{\lambda}=\frac{1}{(2 \pi)^{3}}{ }_{A}\left\langle\boldsymbol{p} \boldsymbol{P}=-\boldsymbol{p}_{\boldsymbol{v}}\left|\left(1+t G_{0}\right) j^{\lambda}\right| \Phi_{\text {deuteron }}\right\rangle \equiv \frac{1}{(2 \pi)^{3}} M^{\lambda}
$$

is the hadronic part. In eq. (1) $\boldsymbol{p}_{\mu}, \boldsymbol{p}_{v}$ are the momenta of the incoming muon (with spin $s_{v}$ ) and outgoing neutrino (with spin $s_{\mu}$ ) and $u, \gamma_{\lambda}$ are Dirac spinors and matrices, respectively. In eq. (2) $\left|\Phi_{\text {deuteron }}\right\rangle$ is the deuteron wave function and ${ }_{A}\left\langle\boldsymbol{p} \boldsymbol{P}=-\boldsymbol{p}_{\boldsymbol{v}}\right|$ is the final anti-symmetrized two nucleon state with the relative momentum of the two neutrons $\boldsymbol{p}$ and total momentum $\boldsymbol{P}=-\boldsymbol{p}_{\boldsymbol{v}}$. Finally $j$ is the weak current operator, $t$ is the two nucleon $(2 \mathrm{~N})$ transition operator and $G_{0}$ is the free propagator.

The crucial matrix elements:

$$
M^{\lambda}={ }_{A}\left\langle\boldsymbol{p} \boldsymbol{P}\left|\left(1+t G_{0}\right) j^{\lambda}\right| \Phi_{\mathrm{d}}\right\rangle={ }_{A}\left\langle\boldsymbol{p} \boldsymbol{P}\left|j^{\lambda}\right| \Phi_{\mathrm{d}}\right\rangle+_{A}\left\langle\boldsymbol{p} \boldsymbol{P}\left|t G_{0} j^{\lambda}\right| \Phi_{\mathrm{d}}\right\rangle
$$

introduced in eq. (2) are a sum of the plane wave and scattering parts. Both contributions can be calculated using the same formalism as was developed in [2] for deuteron electro-disintegration.

Calculation of both the plane wave and scattering parts in (3) require the knowledge of the weak current $j^{\lambda}$. In the present case we limit our selves to single nucleon currents, they are derived using a standard approach from the non relativistic expansion of:

$$
\left\langle\boldsymbol{p}^{\prime}\left|j^{\mu}(1)\right| \boldsymbol{p}\right\rangle=\bar{u}\left(\boldsymbol{p}^{\prime}, s^{\prime}\right)\left(\left(g_{1}^{V}-2 m g_{2}^{V}\right) \gamma^{\mu}+g_{2}^{V}\left(p+p^{\prime}\right)^{\mu}+g_{1}^{A} \gamma^{\mu} \gamma^{5}+g_{2}^{A}\left(p-p^{\prime}\right)^{\mu} \gamma^{5}\right) \tau_{-} u\left(\boldsymbol{p}, s^{\prime}\right)
$$

Where $m$ is the nucleon mass and $\tau_{-}$is the isospin lowering operator. For the results presented in the next section we chose the form factors $g_{1}^{V}, g_{1}^{A}, g_{2}^{V}, g_{2}^{A}$ in (4) to agree with [5].

The scattering part in (3) includes the transition operator $t$ which can be calculated using 3D methods described for example in [1]. The calculation scheme presented there allows for the incorporation of a very general form of the $2 \mathrm{~N}$ potential. In the results presented below we used the Bonn B potential from [6]. 
MESON 2014 $-13^{\text {th }}$ International Workshop on Production, Properties and Interaction of Mesons

\section{Results}

The previous section contains only an outline of the calculation, details are presented in [3]. Here we will demonstrate the final results compared with the classical partial wave approach. Results for the total decay rates for the hyperfine doublet and quadruplet $(f=1 / 2,3 / 2)$ cases calculated using the plane wave part only are compared to the full result:

$$
\begin{array}{cc}
\Gamma_{\text {plane-wave }}^{f=\frac{1}{2}}=363.511[1 / s] & \Gamma_{\text {full }}^{f=\frac{1}{2}}=396.118[1 / s] \\
\Gamma_{\text {plane-wave }}^{f=\frac{3}{2}}=10.425[1 / s] & \Gamma_{\text {full }}^{f=\frac{3}{2}}=12.231[1 / s]
\end{array}
$$

The scattering contribution to the total decay rate is clearly visible and amounts to about $8 \%$ of the full rate. Additionally it can be seen that the quadruplet capture rate is significantly smaller then the doublet rate.

\section{Acknowledgments}

The project was financed from the resources of the National Science Centre (Poland), decision number: DEC-2013/11/N/ST2/03733. Some of the calculations have been performed on the Supercomputing cluster of the JSC, Jülich Germany.

\section{References}

[1] J. Golak et al., Few-Body Syst. 53, 237-252 (2012)

[2] K. Topolnicki et al., Few-Body Syst. 54, 2233-2253 (2013)

[3] J. Golak et al., Phys. Rev. C 90, 024001 (2014)

[4] J.D. Walecka, Theoretical Nuclear and Subnuclear Physics (Oxford University Press, New York, 1995)

[5] L.E. Marcucci et al., Phys. Rev. C 83, 014002 (2011)

[6] R. Machleidt, The Meson Theory of Nuclear Forces and Nuclear Structure (Springer US, 1989) volume 19 